\title{
Global Stability of a Computer Virus Model with Cure and Vertical Transmission
}

\author{
Chunmei Zeng*, Yujiang Liu \\ Key Laboratory of Jiangxi Province for Numerical Simulation and Emulation Techniques, \\ Gannan Normal University, Ganzhou, P.R.China \\ *gnsyzcm@126.com
}

\begin{abstract}
In this paper, an SLBS computer virus propagation model with cure and vertical transmission rate is proposed and analyzed. The basic reproduction number are obtained which guarantee computer virus eradication. Moreover we demonstrate that the viral equilibrium is globally asymptotically stable if the basic reproduction number is more than unity. Some numerical simulations illustrate our main results. According to theory and simulation, a set of policies is proposed for dropping or eradicating computer virus propagation across the Internet effective.
\end{abstract}

Keywords: Computer virus, Vertical transmission, Lyapunov function, Globally asymptotically stable.

\section{INTRODUCTION}

With the development of technology and society, people are increasingly inseparable from the Internet and computer. Simultaneously accompanied by computer viruses problem has become more prominent, and caused serious damages for computer users information security. Consequently, it is urgent us to better understand how computer viruses spread over the Internet and to get effective e measures to cope with this issue.

As the names imply, a computer virus are quite similar with a biological virus in the infectiousness. So a serial of classic epidemicals such as the SIR model [1, 2], SEIR model [3,4], the SEIQV model[5], are proposed. But above all of these more or less neglect the intrinsic difference between the computer virus and biological virus. Consider the fact that the E compartment (latent computers) can infect the other computers, L.X. Yang, X. Yang et al. suggest a series of novel epidemic models, known as the SLBS models and reveal their dynamical behaviors[6-8]. In real computer network, vertical transmission plays an important role in the spread of computer virus [3]. As a result of a new computer on its software installation process may introduces the virus, infectious in nature transmit through both horizontal and vertical modes.

Motivated by the above comment, the present paper addresses a SLBS model with cure and vertical transmission, where some degree of a cure against a computer virus is considered, and it moves into the susceptible class from latent computers or infected computers.

Our paper is organized as follows. In section 2 we formulate the complete mathematical model. Section 3 we define the basic reproductive number and give its biological interpretation.

Moreover, the existence of equilibria is given. The stabilities of the disease free and endemic equilibria of the model are obtained in section 4-5. Section 6 discuss the results and perform some numerical simulations to support these results.

\section{THE MODEL}

Let $\mathrm{S}(\mathrm{t}), \mathrm{L}(\mathrm{t})$ and $\mathrm{B}(\mathrm{t})$ denote the number of computers that it stays in uninfected, latent and breaking-out. For convenient, it denotes with S, L, B, respectively. we consider the following model based on [7].

$\dot{S}=\Lambda-p_{1} L-p_{2} B-\beta_{1} S L-\beta_{2} S B+\gamma_{1} L+\gamma_{2} B-\mu S$

$\dot{L}=\beta_{1} S L+\beta_{2} S B+p_{1} L-\left(\gamma_{1}+\alpha+\mu\right) L$ 
$\dot{B}=\alpha L+p_{2} B-(\gamma 2+\mu) B$

where

$\Lambda$, the computer number is increased via recruiting at a constant rate.

$p_{i}(i=1,2)$, the vertical transmission rate that the recruited computers who are infected by

latent computers ( $\mathrm{p} 1)$ or breaking-out computers $(p 2)$.

$\mu$, the remove rate of computers from network;

$\beta_{i}(i=1,2)$, transmission coefficient of $\mathrm{L}, \mathrm{B}$;

$\gamma_{i}(i=1,2)$, cure rate of latent computers and breaking-out computers;

$\alpha$, the rate that transfer latent computer to breaking-out computers.

It is obvious that the region $\{(S, L, B) \mid S \geq 0, L \geq 0, B \geq 0\}$ positively invariant for the model

(1). Summing up the three equations in model (1), we have

$(S+L+B)^{\prime}=\Lambda-\mu(S+L+B)$

then $\lim \sup _{t \rightarrow \infty}(S+L+B) \leq=^{\Delta} N$ Let $\max \{p 1, p 2\}<\mu$, then we obtain the region

$\Omega=\{(S, L, B) \mid S \geq 0, L \geq 0, B \geq 0, S+L+R \leq N\}$

is a positive invariant set of system (1). We will consider the dynamic behavior of system (1)

on $\Omega$.

Substitute $S=N-L-B$ into the second equation of system (1), we can consider the following twodimensional limiting system:

$\dot{L}=\left(\beta_{1} L+\beta_{2} B\right)(N-L-B)-\left(\gamma_{1}+\alpha+\mu-p_{1}\right) L$

$\dot{B}=\alpha L+p 2 B-(\gamma 2+\mu) B$

within a positive invariant set $\Omega_{2}=\{(L, B) \mid L \geq 0, B \geq 0, L+B \leq N\}$.

\section{THE BASIC REPRODUCTION NUMBER AND EQUILIBRIA}

The basic reproductive number has played a central role in epidemiological theory for infectious diseases because it it provides an index of transmission intensity and establishes threshold criteria. In this section, we will calculate the basic reproduction number of system (1).

It is easy to see that system (1) always has a virus-free equilibrium (the absence of infectious node, that is, $L=0, B=0), E_{0}(N, 0,0)$.

$$
R_{0}=\frac{\beta_{1} N}{\gamma_{1}+\alpha+\mu-p_{1}}+\frac{\beta_{2} \alpha N}{\left(\gamma_{1}+\alpha+\mu-p_{1}\right)\left(\gamma_{2}+\mu-p_{2}\right)}
$$

Let $R_{1}=\frac{\beta_{1} N}{\gamma_{1}+\alpha+\mu-p_{1}}$ and

$$
R_{2}=\frac{\beta_{2} \alpha N}{\left(\gamma_{1}+\alpha+\mu-p_{1}\right)\left(\gamma_{2}+\mu-p_{2}\right)} .
$$

R1 represents the average number of secondary infections generated by an infectious individual through computer nodes who are contaminated by infection during his mean time staying in the latent period, Similarly, R2 represents the average number of secondary infections by an infection who survived undergo latent during his mean time on breaking. The viral equilibrium of system (1), $E^{*}\left(S^{*}\right.$, $\left.L^{*}, B^{*}\right)$, is determined by equations

$\Lambda-p_{1} L-p_{2} B-\beta_{1} S L-\beta_{2} S B+\gamma_{1} L+\gamma_{2} B-\mu S=0$ 
$\beta_{1} S L+\beta_{2} S B+p_{1} L-(\gamma 1+\alpha+\mu) L=0$

$\alpha L+p_{2} B-(\gamma 2+\mu) B=0$

Using the third equation of (4), we have

$$
B=\frac{\alpha}{\gamma_{2}+\mu-p_{2}} L
$$

For, $\mathrm{L} \neq 0$ substituting it into the second equation of (4), we can obtain

$$
S^{*}=\frac{N}{R_{0}}
$$

Substituting (5) into the first equation of (4) yields

$$
L^{*}=\frac{\left(\gamma_{2}+\mu-p_{2}\right) N}{\gamma_{2}+\mu+\alpha-p_{2}}\left(1-\frac{1}{R_{0}}\right)
$$

Furthermore

$$
B^{*}=\frac{\alpha N}{\gamma_{2}+\mu+\alpha-p_{2}}\left(1-\frac{1}{R_{0}}\right)
$$

Theorem 1 System (2.1) has the only infection free equilibrium $E(N, 0,0)$ and also has a unique infection equilibrium $\mathrm{E}^{*}\left(S^{*}, L^{*}, B^{*}\right)$ besides $E_{0}$ for $R_{0}>1$.

\section{THE VIRUS-FREE EQUILIBRIUM AND ITS STABILITY}

A global stability result for the virus-free equilibrium $E_{0}$ of system (1) is given below.

Theorem1. The virus-free equilibrium $E_{0}$ of system (1) is locally asymptotically stable for $R_{0} \leq 1$ and unstable for $R_{0}>1$.

Proof. Let us calculate the Jacobian matrix at $E_{0}$

$$
J_{0}=\left(\begin{array}{cc}
\beta_{1} N-\left(\gamma_{1}+\alpha+\mu-p_{1}\right) & \beta_{2} N \\
\alpha & -\left(\gamma_{2}+\mu-p_{2}\right)
\end{array}\right)
$$

The corresponding characteristic equation of $J_{0}$ is

$$
\lambda^{2}-\operatorname{Tr}\left(J_{0}\right) \lambda+\operatorname{Det}\left(J_{0}\right)=0,
$$

Where

$$
\begin{gathered}
\operatorname{Tr}\left(J_{0}\right)=\beta 1 n-(\gamma 1+\alpha+\mu-p 1)-(\gamma 2+\mu-p 2) \\
=-\left(\gamma_{1}+\alpha+\mu-p 1\right)\left(1-R_{0}\right)-\frac{\alpha \beta 2 N}{\gamma_{2}+\mu-p_{2}} \\
-(\gamma 2+\mu-p 2) \\
\operatorname{Det}(J 0)=-(\beta 1 n-(\gamma 1+\alpha+\mu-p 1))(\gamma 2+\mu-p 2) \\
-\alpha \beta 2 N \\
=(\gamma 1+\alpha+\mu-p 1)(\gamma 2+\mu-p 2)(1-R 0)
\end{gathered}
$$

If $R_{0}<1$, we have $\operatorname{Tr}\left(J_{0}\right)<0, \operatorname{Det}\left(J_{0}\right)>0$ It follows that the two roots of the equation (8) have negative real parts. By the Hurwitz criterion, $E_{0}$ is locally asymptotically stable.

If $R_{0}>1$, we have $\operatorname{Det}\left(J_{0}\right)<0$. It mean that the equation (8) have two roots $\lambda_{1}>0, \lambda_{2}<0$, $E_{0}$ is unstable. The proof is complete. 
Theorem2. The virus-free equilibrium $E_{0}$ of system (1) is globally asymptotically stable for $R_{0} \leq 1$.

Proof. Define a Lyapunov function

$V(L, B)=\frac{L_{2}+a B_{2}}{2}$

Then derivative of the function $V(L, B)$ with respected to $t$ along an orbit of system (2) is given by

$$
\begin{aligned}
\left.\frac{d V}{d t}\right|_{(2)}= & \beta 1 S L^{2}+\beta 2 S B L-(\gamma 1+\alpha+\mu-p 1) L^{2}+a \alpha L B \\
& -a\left(\gamma_{2}+\mu-p 2\right) B^{2} \\
\leq & \beta 1 N L^{2}+\beta 2 N B L-(\gamma 1+\alpha+\mu-p 1) L^{2}+a \alpha L B \\
& -(\gamma 2+\mu-p 2) B^{2} \\
= & \left(\beta 1 N-R_{0}(\gamma 1+\alpha+\mu-p 1)\right) L^{2}+(\beta 2 N+a \alpha) L B \\
& -a(\gamma 2+\mu-p 2) B^{2}-(1-R 0)(\gamma 1+\alpha+\mu-p 1) L^{2} \\
= & \frac{\beta_{2} N_{\alpha}}{\gamma_{2}+\mu-p_{2}}+\left(\beta_{2} N+\alpha \alpha\right) L B \\
- & a\left(\gamma_{2}+\mu-p_{2}\right) B^{2}-\left(1-R_{0}\right)\left(\gamma_{1}+\alpha+\mu-p_{1}\right) L^{2} \\
= & -\left(\sqrt{\frac{\beta_{2} N_{\alpha}}{\gamma_{2}+\mu-p_{2}}} L-\sqrt{a\left(\gamma_{2}+\mu-p_{2}\right) B}\right)^{2} \\
& -\left(2 \sqrt{\alpha \beta_{2} N \alpha}-\left(\beta_{2} N+\alpha \alpha\right)\right) L B \\
& -\left(1-R_{0}\right)\left(\gamma_{1}+\alpha+\mu-p_{1}\right) L^{2}
\end{aligned}
$$

Let $a=\frac{\beta_{2} N}{\alpha}$, then

$$
\begin{aligned}
\left.\frac{d V}{d t}\right|_{(2)}= & -\left(\sqrt{\frac{\beta_{2} N \alpha}{\gamma_{2}+\mu-p_{2}}} L-\sqrt{a\left(\gamma_{2}+\mu-p_{2}\right)} B\right)^{2} \\
& -\left(1-R_{0}\right)\left(\gamma_{1}+\alpha+\mu-p_{1}\right) L^{2}
\end{aligned}
$$

Because $R_{0} \leq 1$, It can be seen that $\left.\frac{d V}{d t}\right|_{(2)} \leq 0$ holds for all $(L, B) \in \Omega_{2}$. Furthermore, the maximum invariant set in $\left\{(\mathrm{L}, \mathrm{B}) \in \Omega_{2},\left.\frac{d V}{d t}\right|_{(2)}=0\right\}$ is the singleton $\{\mathrm{E} 0\}$, therefore, by LasalleLyapunov theorem, every solution that starts in $\Omega_{2}$ approaches $E_{0}$ as $t \rightarrow \infty$. The proof is complete.

\section{The VIRAL EQUILIBRIUM AND ITS STABILITY}

In this section, we study the stability of the viral equilibrium. First we have

Theorem 3. If $R_{0}>1$, the virus-free equilibrium $E_{0}$ of system (1) is locally asymptotically stable.

Proof. the Jacobian matrix at $E^{*}$

$$
\begin{aligned}
& J^{*}= \\
& \left(\begin{array}{cc}
\beta_{1} N-\left(\gamma_{1}+\alpha+\mu-p_{1}\right) & \beta_{2} N-2 \beta_{2} B^{*} \\
-\left(2 \beta_{1} L^{*}+\left(\beta_{1}+\beta_{2}\right) B^{*}\right) & -\left(\beta_{1}+\beta_{2}\right) L^{*} \\
\alpha & -\left(\gamma_{2}+\mu-p_{2}\right)
\end{array}\right)
\end{aligned}
$$


We have

$$
\begin{aligned}
\operatorname{Tr}\left(J^{*}\right)= & \beta_{1} N-\left(\gamma_{1}+\alpha+\mu-p_{1}\right) \\
& -\left(2 \beta_{1} L^{*}+\left(\beta_{1}+\beta_{2}\right) B^{*}\right)-\left(\gamma_{2}+\mu-p_{2}\right) \\
= & \beta_{1} S^{*}-\left(\beta_{1} S^{*}+\frac{\beta_{2} S^{*} B^{*}}{L *}\right)-\left(\beta_{1} L^{*}+\beta_{2} B^{*}\right) \\
& -\left(\gamma_{2}+\mu-p_{2}\right) \\
= & -\frac{\beta_{2} S^{*} B^{*}}{L *}-\left(\beta_{1} L^{*}+\beta_{2} B^{*}\right)-\left(\gamma_{2}+\mu-p_{2}\right) \\
< & 0
\end{aligned}
$$

$$
\begin{aligned}
\operatorname{Det}\left(J^{*}\right)= & -\beta_{1} N\left(\gamma_{2}+\mu-p_{2}\right) \\
& +\left(\gamma_{1}+\alpha+\mu-p_{1}\right)\left(\gamma_{2}+\mu-p_{2}\right) \\
& +\left(2 \beta_{1} L^{*}+\left(\beta_{1}+\beta_{2}\right) B^{*}\right)\left(\gamma_{2}+\mu-p_{2}\right) \\
& +\alpha\left(2 \beta_{2} B^{*}+\left(\beta_{1}+\beta_{2}\right) L^{*}\right) \\
= & -\beta_{1} S^{*}\left(\gamma_{2}+\mu-p_{2}\right)-\beta_{2} \alpha S^{*} \\
& +\left(\gamma_{1}+\alpha+\mu-p_{1}\right)\left(\gamma_{2}+\mu-p_{2}\right) \\
& +(b+\alpha)\left(\beta_{1} L^{*}+\beta_{2} B^{*}\right)
\end{aligned}
$$

From the second and the third equation of system (1). we have

$$
\begin{aligned}
& \left(\gamma_{1}+\alpha+\mu-p_{1}\right)\left(\gamma_{2}+\mu-p_{2}\right) \\
& =\frac{\beta_{1} S^{*} L^{*} \alpha}{B}+\beta_{2} \alpha S^{*} \\
& =\beta_{1} S^{*}\left(\gamma_{2}+\mu-p_{2}\right)+\beta_{2} \alpha S^{*}
\end{aligned}
$$

Substituting (13) into (12), we have

$$
\begin{aligned}
\operatorname{Det}\left(J^{*}\right) & =(b+\alpha)\left(\beta_{1} L^{*}+\beta_{2} B^{*}\right) \\
& >0
\end{aligned}
$$

Based on Routh-Hurwitz Theorem, $E^{*}$ is locally asymptotically stable.

Now, we study the global stability of the viral steady state $E^{*}$. First of all, we consider the

following two lemmas, which are useful in the follows. Let $\Omega^{\prime}=\Omega_{2} 2-E_{0}, \Omega_{0}=\{(L, B) \mid L>$ $\left.0, B>0,(L, B) \in \Omega^{\prime}\right\}, \partial \Omega_{0}=\Omega^{\prime} \mid \Omega_{0}$.

Lemma1. System (2) has no periodic obit on $\Omega_{0}$.

Proof. Note that

$$
\begin{aligned}
& P(L, B)=(\beta 1 L+\beta 2 B)(N-L-B)-(\gamma 1+\alpha+\mu-p 1) L \\
& Q(L, B)=\alpha L+p 2 B-(\gamma 2+\mu) B
\end{aligned}
$$

Let us consider the following Dulac function

$$
D(L, B)=\frac{1}{L B}
$$

Then

$$
\begin{aligned}
\frac{\partial D P}{\partial L}+\frac{\partial D Q}{\partial B} & =-\frac{\beta_{2} N}{L^{2}}-\left(\frac{\beta_{1}}{B}-\frac{\beta_{2} B}{L^{2}}\right)-\frac{\alpha}{B^{2}} \\
& =-\frac{\beta_{2}(N-B)}{L^{2}}-\frac{\beta_{1}}{B}-\frac{\alpha}{B^{2}} \\
& <0
\end{aligned}
$$

Thus, from Bendixson-Dulac criterion that system (1) has no periodic orbit in $\Omega_{0}$. 
Lemma2. System (2) has no periodic obit that goes across a point on $\partial \Omega_{0}$.

Proof. Suppose that $(\tilde{L}, \tilde{B})$ is an arbitrary point on $\partial \Omega_{0}$. Following we will consider three possible cases in term of $\partial \Omega_{0}$.

Case I. $0<\tilde{L} \leq N, \tilde{B}=0$. Then $\left.\dot{B}\right|_{(\tilde{L}, \tilde{B})}=\alpha \tilde{L}>0$.

Case II. $0<\tilde{B} \leq N, \tilde{L}=0$. Then $\dot{L}(\tilde{L}, \tilde{B})=\beta 2 B(N-B)>0$.

Case III. $\tilde{L}+\tilde{B}=N, \tilde{B}=0$. Then $(\dot{L}+\dot{B}) \mid(\tilde{L}, \tilde{B})=-\left(\gamma_{1}+\mu-p 1\right) L-\left(\gamma_{2}+\mu-p 2\right) B<0$.

Hence, the claimed result from the above discussion.

Theorem4. The viral equilibrium $E^{*}$ of system (1) is globally asymptotically stable on $\Omega^{\prime}$ if 2 $R_{0}>1$.

Proof. The claimed result follows by combining Lemma (1), (2) and the Poincare-Bendixson theorem.

\section{NuMerical RESUlts}

We shall verify the validity of the proposed model through numerical simulations. We set $N=500, p 1=0.15, p_{2}=0.23, \beta_{1}=0.002, \beta_{2}=0.005, \gamma_{1}=0.2, \gamma_{2}=0.4, \mu=1.2, \alpha=0.8$, then system (1) becomes:

$$
\begin{gathered}
\dot{S}=416.667-0.15 L-0.23 B-0.002 S L-0.005 S B \\
+0.2 L+0.4 B-1.2 S \\
\dot{L}=0.002 S L+0.005 S B+0.15 L-(0.2+0.8+1.2) L \\
\dot{B}=0.8 L+0.23 B-(0.4+1.2) B(18)
\end{gathered}
$$

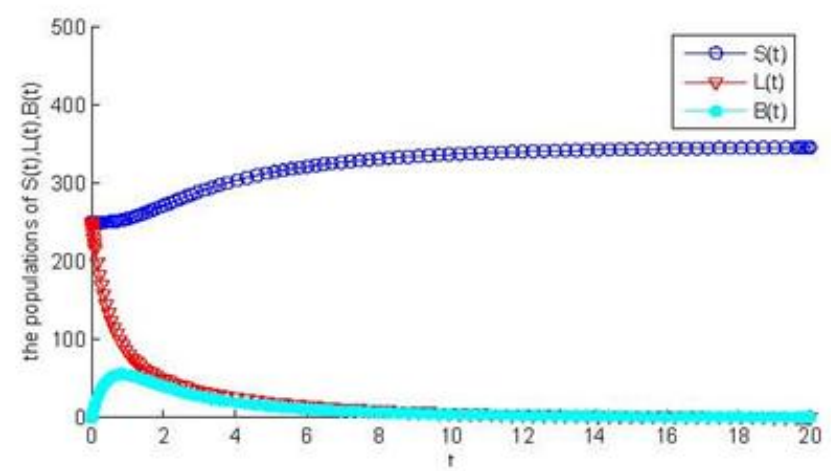

Figure1. Time series of system (18), $R_{0}=0.8333$. The computer virus dies out.

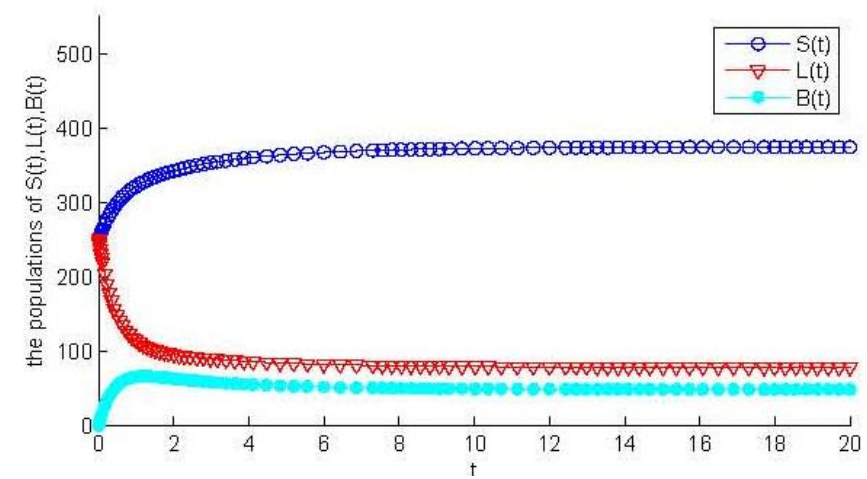

Figure2. Time series of system (1), $p_{1}=p_{2}=0.1, \mu=1$, the other parameters are same as in model (18) $R_{0}=$ 1.3360. The computer virus permanent. 
From (3), $R_{0}=0.8333<1$. According to Theorem $2, E_{0}$ is globally asymptotically stable. We know that the computer virus will die out (see Fig.1). Let $p_{1}=p_{2}=0.1, \mu=1$, then $R_{0}=$

$1.3360>1$. According to Theorem $4, E^{*}$ is globally asymptotically stable and that the computer virus permanent.

Fig. 3 and Fig. 4 show that the tendency of the infected nodes $(S+B)$ with $\beta_{1}, \beta_{2}$. It shows that $L+$ $B$ goes to zero as $\beta_{1}=0.001$ (Fig.3), $\beta_{2}=0.001,0.003$ (Fig.4) for which $R_{0}<1$. Furthermore, we can observe that the value of $L+B$ increases with $\beta_{1}, \beta_{2}$.

The contour plots of Fig.5 shows the dependence of $R_{0}$ on $p_{1}$ and $p_{2}$, and it shows that $R_{0}$ increases more quickly with $p_{1}$ and $p_{2}$. It suggest us that we should pay more attention to software system's installation of introducing virus. Fig. 6 shows the dependence of $R_{0}$ on $\gamma_{1}$ and $\gamma_{2}$. We can observe that it gains a good effective to increase the recovery rate $\gamma_{1}, \gamma_{2}$ when $\gamma_{1}$ or $\gamma_{2}$ is very small.

The plotyy of Fig.7 shows that the $R_{0}, B$ and $L+B$ increase as $\alpha$ increases, Fig. 8 shows that

the $R_{0}, B$ and $L+B$ decrease rapidly as $\mu$ increases, and when $\mu=\mu^{*}=1.4132, R_{0}\left(\mu^{*}\right)=1$. If $\mu<\mu^{*}$, $R_{0}(\mu)<1$ and $B_{\infty}=0$ and $L_{\infty}+B_{\infty}=0$. It prompt that

\section{CONClusion}

In this paper, we have analyzed an SLBS model with vertical transmission and we consider the L compartment and B compartment infective rate are distinct. We have studied the stability of virus-free equilibrium. We find that the virus-free equilibrium is globally asymptotically stable under the basic reproduction number and viral equilibrium is globally asymptotically

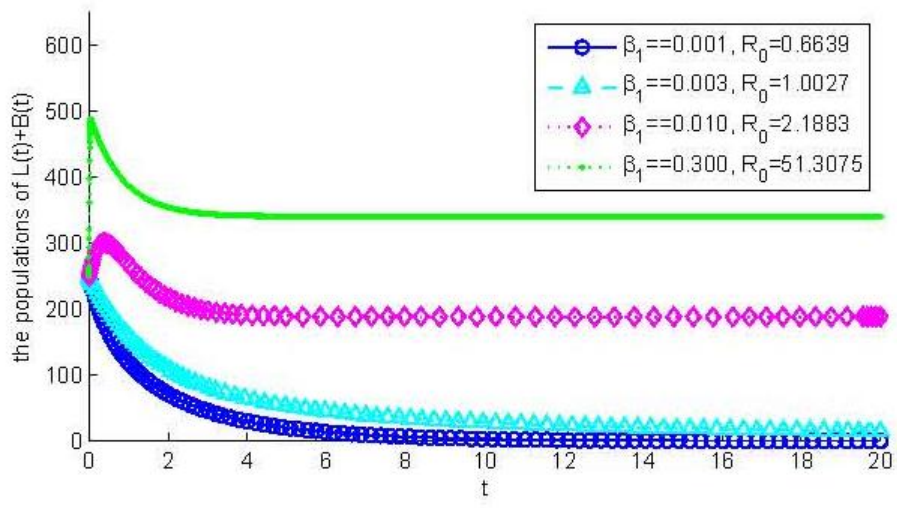

Figure3. The tendency of the infected nodes $(L(t)+B(t))$ with different value of $\beta_{1}($ all other parameters are same as in model (18) except for $p_{1}=p_{2}=0.1, \mu=1$ ).

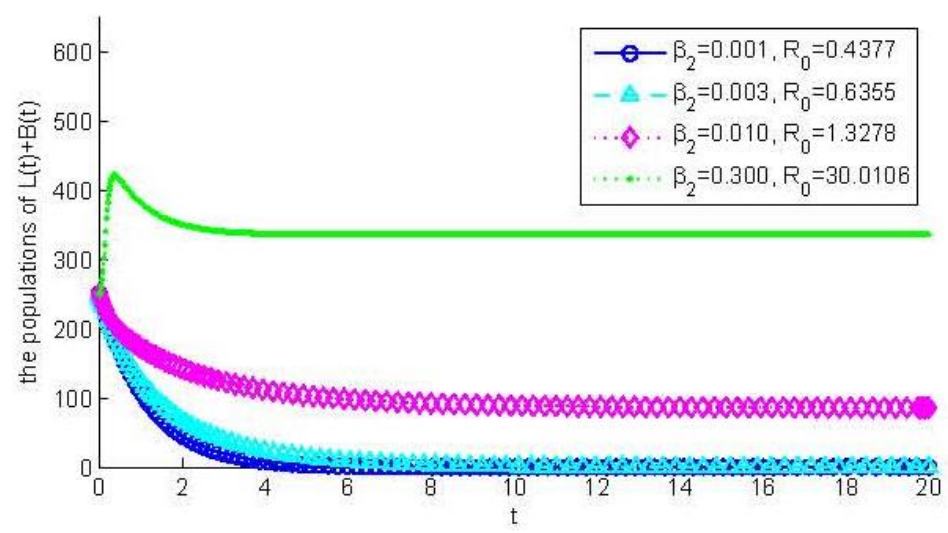

Figure4. The tendency of the infected nodes $(L(t)+B(t))$ with different value of $\beta_{2}($ all other parameters are same as in model (18) except for $p_{1}=p_{2}=0.1, \mu=1$ ). 


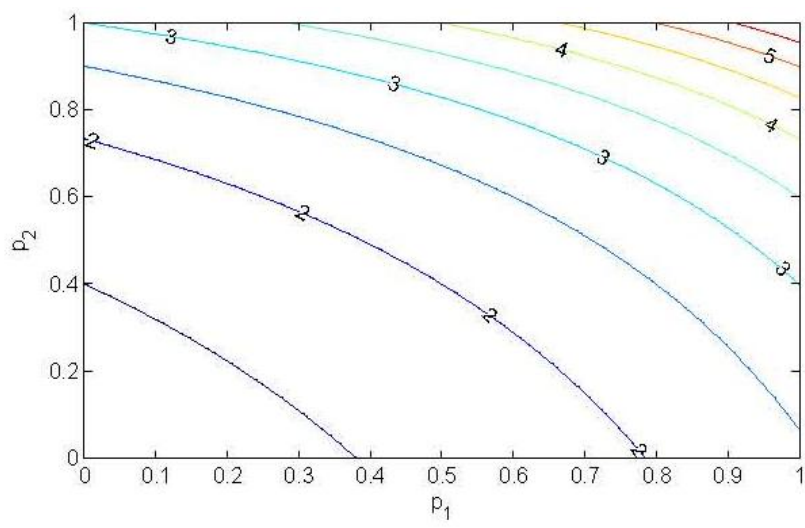

Figure5. Contour plots of $R_{0}$ versus the vertical transmission rate $p_{1}, p_{2}($ all other parameters are same as in model (18) except for $\left.p_{1}=p_{2}=0.1, \mu=1\right)$.

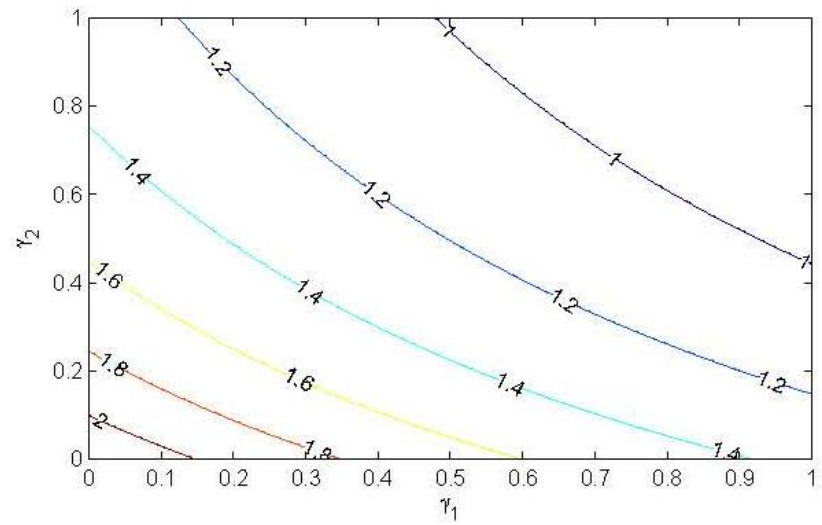

Figure6. Plot contours of $R_{0}$ versus recover rate $\gamma_{1}, \gamma_{2}($ all other parameters are same as in model (18) except for $\left.p_{1}=p_{2}=0.1, \mu=1\right)$.

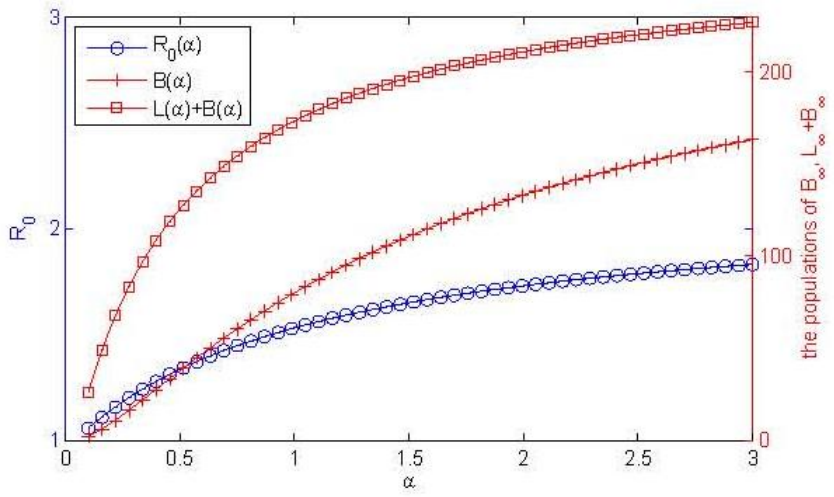

Figure7. Plots of $R_{0}$ and $L_{\infty}+B_{\infty}$ with $\alpha$ (all other parameters are same as in model (18) except for $p_{1}=p_{2}=$ $0.1, \mu=1)$.

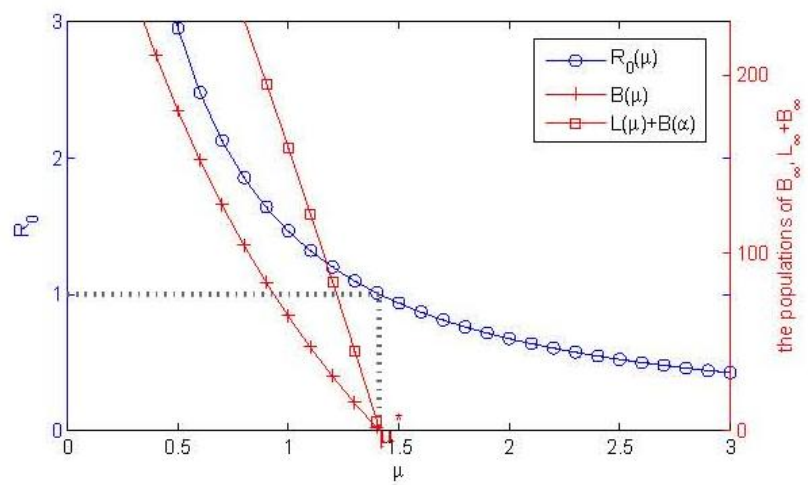

Figure8. Plots of $R_{0}$ and $L_{\infty}+B_{\infty}$ with $\mu$ (all other parameters are same as in model (18) except for $p_{1}=p_{2}=$ $0.1, \mu=1), \mu^{*}=1.4132$. 
stable otherwise. The analysis and numerical simulation of the SLBS model shows that the introduction of vertical transmission modifies the threshold of system for an epidemic to occur. It is important to emphasize that the factors of computer virus propagation always changes over time. we leave this topic for future work.

\section{ACKNOWLEDGMENT}

The research has been supported by the 12th Five-year Education Scientific Planning Project of Jiangxi (15ZD3LYB031) and the Social Science Planning Projects of Jiangxi Province (14XW08).

\section{REFERENCES}

[1] M. Youssef and C. Scoglio, "An individual-based approach to sir epidemics in contact networks," Journal of theoretical biology, vol. 283, no. 1, pp. 136-144, 2011.

[2] J. Ren, Y. Xu, Y. Zhang, Y. Dong, and G. Hao, "Dynamics of a delay-varying computer virus propagation model," Discrete Dynamics in Nature and Society, vol. 2012, 2012.

[3] B. K. Mishra and S. K. Pandey, "Dynamic model of worms with vertical transmission in computer network," Applied Mathematics and Computation, vol. 217, no. 21, pp. 8438-8446, 2011.

[4] T. Dong, X. Liao, and H. Li, "Stability and hopf bifurcation in a computer virus model with multistate antivirus," in Abstract and Applied Analysis, vol. 2012. Hindawi Publishing Corporation, 2012.

[5] F. Wang, Y. Zhang, C. Wang, J. Ma, and S. Moon, "Stability analysis of a seiqv epidemic model for rapid spreading worms," Computers \& Security, vol. 29, no. 4, pp. 410-418,2010.

[6] L.-X. Yang, X. Yang, L. Wen, and J. Liu, "A novel computer virus propagation model and its dynamics," International Journal of Computer Mathematics, vol. 89, no. 17, pp. 2307-2314, 2012.

[7] L.-X. Yang, X. Yang, Q. Zhu, and L. Wen, "A computer virus model with graded cure rates," Nonlinear Analysis: Real World Applications, vol. 14, no. 1, pp. 414-422, 2013.

[8] L.-X. Yang and X. Yang, "Propagation behavior of virus codes in the situation that infected computers are connected to the internet with positive probability," Discrete Dynamics in Nature and Society, vol. 2012, 2012. 